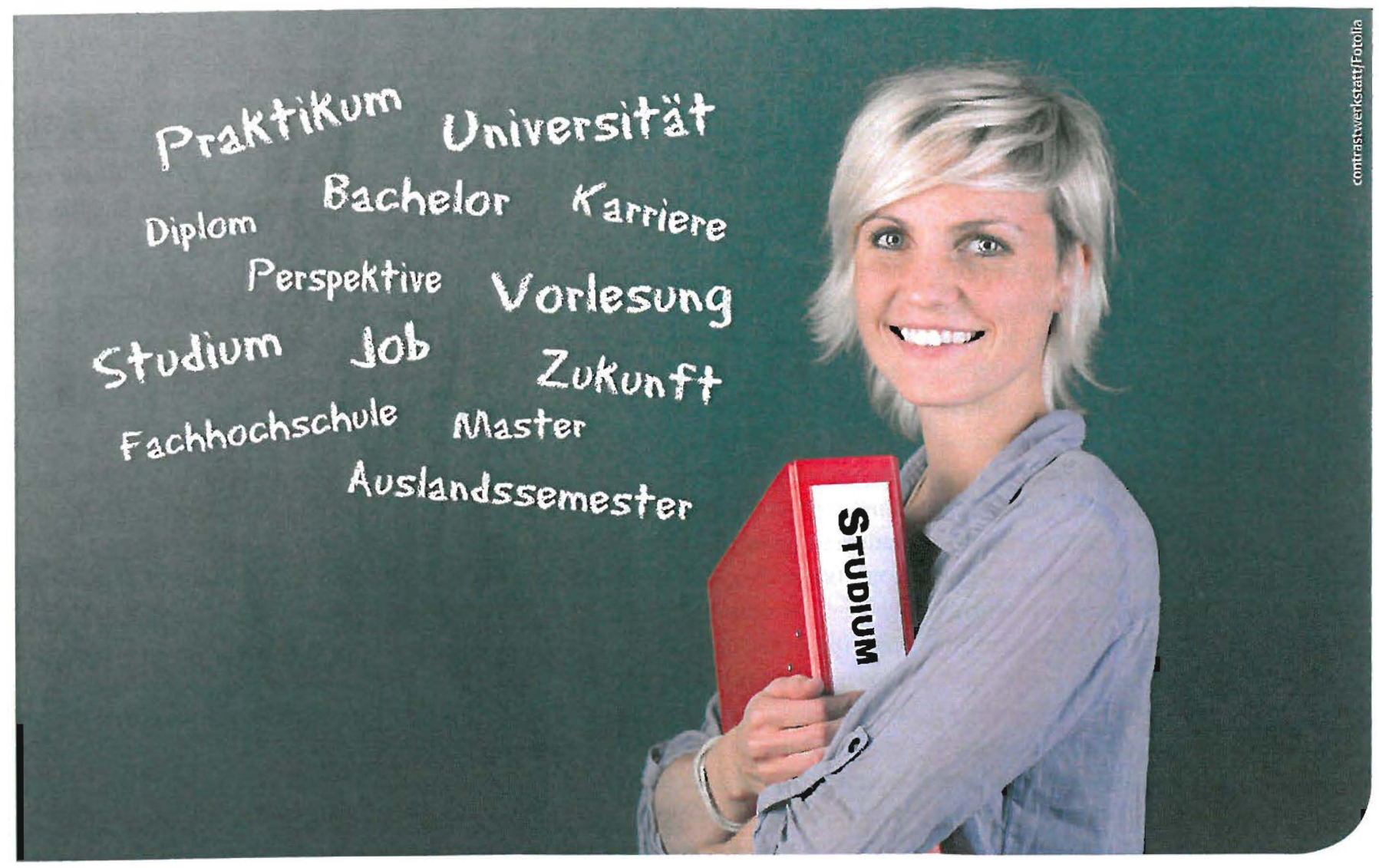

ADVCANCED PRACTICE NURSING (APN)

\title{
Work-Shadowing in den USA
}

\section{Daniela Händler-Schuster und Romy Mahrer-Imhof}

Ein Work-Shadowing ist eine Art Praktikum im englischsprachigen Ausland; Pflegefachpersonen können in ihrer Praxis begleitet werden, „Workshadows" können Fragen stellen und Eindrücke erhalten. Ziel ist, die eigene Pflegepraxis zu reflektieren und eine Standortbestimmung vorzunehmen. Die Autorinnen beschreiben, wie sie das APN in den USA erlebt haben und wie eine APN-Rolle in die Praxis implementiert werden kann.

Der vorliegende Beitrag folgt der Frage, wie sich die Rollen im Konzept des Advanced Practice Nursing unterscheiden und berücksichtigt dreì Schwerpunkte.

1. Erstens wird die vertiefte Pflegepraxis (A.PN = Advanced Practice Nursing) an Beispielen persönlicher Erfahrungen beschrieben, die mithilfe eines WorkShadowings in einem Krankenhaus in San Francisco, Kalifornien, gemacht werden konnten, sowie anhand von Erkenntnissen aus einem Interview mit einer Clinical Nurse Specialist (CNS) an der Universität von Kalifornien in San Francisco (UCSF).
2. Zweitens werden aufgrund der persönlichen Erfahrungen und mithilfe eines Literaturvergleichs Wege beschrieben, die auf der praktischen Ebene zu einer gelingenden Rollenfindung beitragen können.

3. Drittens werden Möglichkeiten aufgezeigt, sich für eine vertiefte Pflegepraxis im Master of Science in Pflege vorzubereiten.

Der vorliegende Bericht zeigt ferner, dass Bestrebungen für eine APN-Praxis mittlerweile auch im deutschsprachigen Raum verbreitet sind, was sich an dem neuen Master of Science Studiengang der Fach- hochschulen in der Schweiz zeigt, der auf APN-Praxis fokussiert.

\section{Austausch bildet}

Als Pflegende mit Schwerpunkt gerontologische Langzeitpflege beendete ich im Jahr 1993 meine dreijährige Grundausbildung und war anschließend zehn Jahre sowohl in der heimbezogenen als auch in der häuslichen Pflegepraxis tätig. Es folgte ein Studium in Pflegepädagogik und in Gesundheits- und Pflegewissenschaft. Mit dem Wechsel von der Praxis in die Lehre im Jahr 2003 begegnete mir der Begriff "APN" (Advanced Practice Nursing) im 
deutschsprachigen Raum immer häufiger. Persönlich habe ich mir häufig die Frage gestellt, wie die Rollen einer vertieften Pflegepraxis im Ausland gelebt werden und welche Herausforderung das Hineinwachsen in eine solche Rolle darstellt. Als ich meine neue Stelle als Dozentin an der Zürcher Hochschule für Angewandte Wissenschaften (ZHAW) im Studiengang Master of Science in Pflege antrat, besuchte ich für die Beantwortung meiner Frage und für das Kennenlernen eines anderen Pflegeund Ausbildungssystems zwei Wochen die School of Nursing an der University of California, San Francisco (UCSF), Kalifornien. In diesem Zusammenhang nutzte ich den Austausch mit Fachpersonen, die in der Pflegebildung nach der Grundausbildung für eine erweiterte Pflegepraxis tätig sind. Ich besuchte Seminare, führte Gespräche mit Personen aus unterschiedlichen Disziplinen der Pflegepraxis und absolvierte ein Work-Shadowing bei einer Pflegenden mit APN im Laguna Honda Hospital and Rehabilitation Centers in San Francisco.

\section{Advanced Practice Nursing (APN)}

Nach Hamric, Spross und Hanson (2009) steht Advanced Praxtice Nursing für ein Konzept einer erweiterten, wirksamen Pflege- und Betreuungspraxis. Zu der Rolle einer APN-Pflegenden gehört - neben der erweiterten klinischen Praxis - die Analyse und Synthese des evidenzbasierten Pflegewissens, das Verstehen, das Anwenden von Forschung und Theorie und das Generieren von neuem Wissen. Die klinische Pflegepraxis fokussiert dabei eine Patientengruppe und bezieht das familiäre Setting mit ein.

\section{Kompetenzen und Rollen im APN-Konzept}

Nach Hamric et al. (2009) werden sechs Kernkompetenzen einer vertieften Pflegepraxis unterschieden: Einerseits „Expertencoaching“, „Beratung " und „Forschung", andererseits „Klinisches Leadership“, „Zusammenarbeit" sowie "Klinische Entscheidungsfindung".

In den USA werden vier Rollen unterschieden, die jeweils mindestens einen Master of Science in Pflege erfordern und sich innerhalb der Rolle auf einen Spezial- bereich der Pflegepraxis fokussieren. Diese Rollen sind:

1. "Nurse practitioner" (NP),

2. "Clinical nurse specialist" (CNS),

3. "Nurse anesthetist" (NA) und

4. „Nurse midwive“ (NM).

Ein Beispiel für ein Spezialbereich der Pflegepraxis ist beispielsweise die/der Nurse practitioner mit Fokussierung auf die gerontologische Pflegepraxis (Hamric et al. 2009). Da ich mit meinem WorkShadowing vor allem die Rollenunterschiede zwischen Nurse Practitioners und Clinical Nurse Specialists kennenlernen wollte, werde ich mich in den folgenden Ausführungen auf diese beiden Rollen beschränken und im ersten Teil des folgenden Kapitels einen Kurzüberblick über die Historie der Entwicklung beider Rollen berichten.

\section{Clinical Nurse Specialist (CNS) und Nurse Practitioner (NP)}

Im Amerika reichen die Anfänge einer Rollenbildung einer APN bis in die erste Hälfte des 20. Jahrhunderts zurück. Erstmalig wurde die Rolle einer CNS von Hildegard Peplau in den frühen 1940er-Jahren beschrieben (Peplau 1965). Das erste CNSProgramm folgte im Jahr 1954 für die psychiatrische Pflege (Keeling 2009). Das erste NP-Programm entstand erst etwas mehr als zehn Jahre später, also 1965 im Rahmen der pädiatrischen Pflege. Eine CNS hat unterstützende Komponenten in ihrer Rolle, wozu sowohl das klinische Leadership wie auch Beratung, Lehre und Forschung gehören. Sie erarbeitet im klinischen Setting Lösungen und führt für Familien, Gruppen und Gemeinschaften eine präventive Gesundheitsförderung durch (Delamaire u. Lafortune 2010). Die NP findet ihren Hauptaufgabenbereich in der direkten Pflegepraxis, das heißt, in der Patientenbetreuung unter Einbezug der Familie. Sie verordnet z.B. Medikamente, führt Körperuntersuchungen durch und interpretiert Tests, die sie selbst durchführt (Canadian Nurses Association 2008). Im Vergleich zur CNS (2,5\%) ist die NP wesentlich verbreiteter, nämlich $6,5 \%$ aller registrierten Pflegenden (Delamaire $u$. Lafortune 2010). Die NP wird häufig in der Grundversorgung vor allem in ländlichen Gegenden eingesetzt.

\section{Das Work-Shadowing}

Mit dem zweitägigen Work-Shadowing in einem Krankenhaus begleitete ich eine APN-Pflegende in ihrem Aufgaben- und Verantwortungsfeld. Dazu gehörten neben der direkten pflegerischen Praxis, die Begleitung von Angehörigen und die Beratung zu pflegespezifischen Versorgungsfragen. Ebenso gehörten zu ihren Aufgaben und Verantwortungsfeld die praktische Integration von Forschungsergebnissen, sowie die fallbezogene Zusammenarbeit mit anderen Berufsgruppen, wozu die eigenständige Entscheidungsfindung zählte.

\section{Besuch eines Krankenhauses mit Schwerpunkt Rehabilitation}

Das Laguna Honda Hospital \& Rehabilitation Center in San Francisco unterstützt mit seinen ca. 1400 Mitarbeitern die Bewohner darin, durch Unfall, Krankheit oder Behinderung verlorene Fähigkeiten wieder zu erlangen. Die Pflegende, eine APN-Pflegende mit eínem Schwerpunkt in der gerontologischen Pflege, spezialisierte sich während ihrer beruflichen Entwicklung auf die Palliativpflege. Sie bezeichnete sich als APNPflegende, weil sie ihren Aufgaben- und Verantwortungsbereich nicht klar'zwischen der Rolle einer CNS und NP unterscheiden konnte. Neben den anderen Pflegenden, die mehrheitlich über eine allgemeine Berufsqualifikation in der Pflege verfügten, war sie - neben einer Studierenden im Master of Science in Pflege - die einzige mit APNSpezialisierung und einem PhD (Doktorat).

In den ersten Gesprächen berichtete sie von ihrer täglichen Arbeit in der direkten Pflegepraxis; dabei schilderte sie sowohl von Erfahrungen in der Beziehung mit Bewohnern, als auch mit deren Familienangehörigen. Sie berichtete mir von der täglichen Pflegepraxis auf den jeweiligen Abteilungen und erläuterte mir die Funktion der Pflegenden auf der Abteilung, die ich während ihrer Arbeit außerhalb der Bewohnerzimmer beobachten konnte. Ähnlich wie in einer Wohngruppe hielten sich die Bewohner in einem großen Raum auf, in dem auch Freizeitaktivitäten durchgeführt wurden. Gleichzeitig war der Arbeitsplatz der Pflegenden, wo die schriftliche Pflegearbeit erfolgte, offen, also mitten in diesem Raum platziert, wohingegen die Medikamente separat in anderen 
Räumen aufbewahrt wurden. Kleinere Räume boten die Möglichkeit für getrenntes Essen der Bewohner oder für andere Arbeiten der Mitarbeiter, die Ruhe erforderten. Aus einer Jukebox, die von den Bewohnern selbst bedient wurde, ertönte Musik. Es lief Marvin Gaye. Ein Mitarbeiter sang mit, während sie einer Bewohnerin das Essen gab.

Während unseres Rundgangs zeigte mir die APN-Pflegende u.a. eine Abteilung, auf der ausschließlich chinesische Patienten von chinesischen Mitarbeitern pflegerisch begleitet und versorgt wurden. Ich sah, dass die interkulturelle Pflege einen großen Stellenwert einnahm. Die Mitarbeiter hatten mehrheitlich eine chinesische Herkunft, was sich vor allem in der Umgebungsgestaltung, in der Sprache und auch in den Mahlzeiten zeigte. Nach dem Rundgang begleitete ich die APN-Pflegende zu einer Frau, mit der sie ein Assessment durchführte, das sie zuvor am Computer vorbereitete. Ich konnte beobachten, dass sie zu der Bewohnerin in der Kommunikation ein partnerschaftliches Verhältnis pflegte und mit ihr die einzelnen Schritte des Assessments in meinem Beisein abstimmte. AnschlieBend folgte ich ihr zu einer interdisziplinären Fallbesprechung, bei der insgesamt neun Personen anwesend waren. An dieser Sitzung fanden drei Gespräche mit insgesamt vier Angehörigen von Patienten statt. Anhand der Dokumentation, die im Vorfeld besprochen wurde, wurden die Ergebnisse der Assessments diskutiert und EmpfehIungen abgeleitet. Anschließend wurde im direkten Austausch mit den Angehörigen das weitere Vorgehen vereinbart.

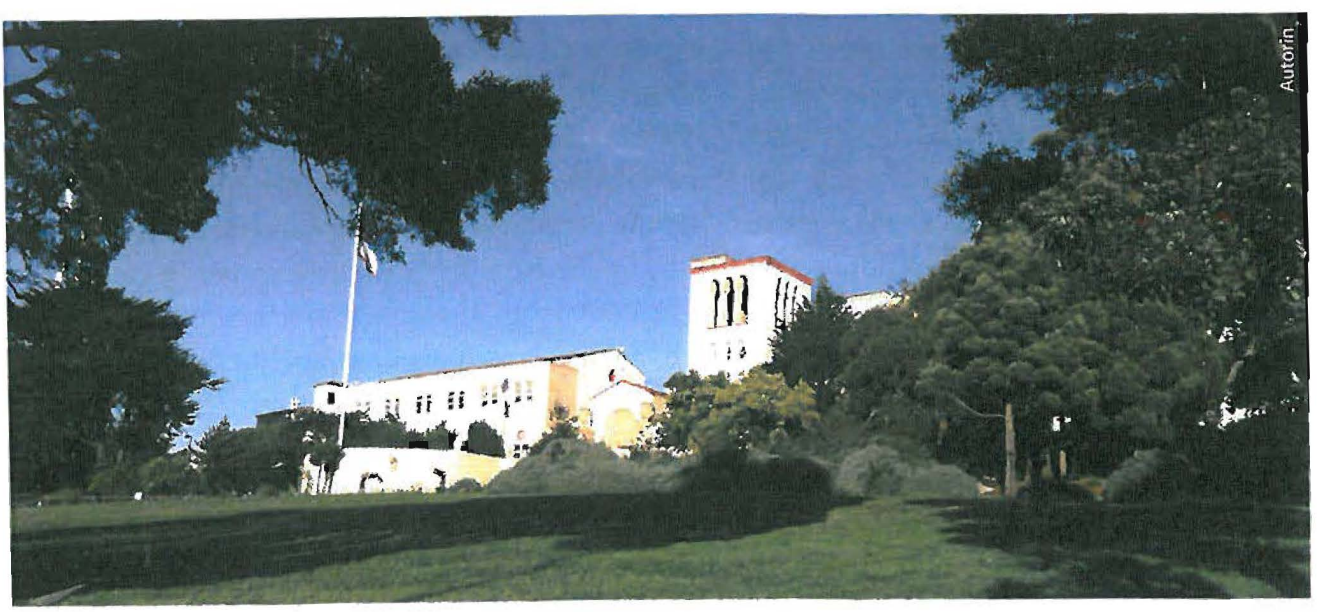

Abb. 5 Das Laguna Honda Hospital \& Rehabilitation Center in San Francisco.
Ich erlebte sehr positiv, dass sich die Mitglieder den Angehörigen der Bewohner mit Namen und Funktion vorstellten. Ebenfalls konnte ich beobachten, wie sie die Zusammenarbeit mit anderen Berufsgruppen gestaltete. Die anderen Mitarbeiter sprachen aus ihrer Sicht zur Situation, so fragte beispielsweise der Seelsorger nach der spirituellen Bedeutung für den Bewohner im Umgang mit der jeweiligen Erkrankung. Der Sozialarbeiter erkundigte sich nach der familiären Situation im häuslichen Umfeld. Die APN fragte die Angehörigen: „Was, glauben Sie, ist das schwierigste für Ihren Vater in der Krebserkrankung angesichts der Gewissheit, dass er sterben muss?" Alle Beteiligten hörten aufmerksam zu und versuchten über weitere Fragen auch ihren persönlichen Unterstützungsbedarf herauszufinden. Die Gespräche waren zum Teil sehr emotional, was sich beispielsweise an der Trauer der Angehörigen zeigte, wenn sie über die belastenden Momente in der Begleitung ihrer kranken Familienangehörigen sprachen.

Nach dieser Sitzung begleitete ich die APN-Pflegende zu einer sehr jungen, sterbenden Frau. Während ich vor dem Zimmer stand, informierte sie die junge Frau über meine Anwesenheit und fragte, ob ich mit ins Zimmer dürfe. Nach ihrem "Ja" betrat auch ich das Zimmer. Am Bett der jungen Frau saß ihre Schwester. Ich konnte beobachten, dass die APN-Pflegende ein partnerschaftliches Verhältnis sowohl zu der kranken Frau als auch zu der Schwester pflegte, was sich durch die vertraute Kommunikation und das Eingehen auf die
Bedürfnisse zeigte. Anschließend folgte ich ihr zu den administrativen Aufgaben im Rahmen der pflegerischen Versorgung dieser Patientin. Sie beantwortete E-Mails, füllte Formulare aus und sprach sich mit den Mitarbeitern ab. Die Angehörige der Bewohnerin suchte die APN-Pflegende nochmals auf, um ihr eine Information zu überbringen, die sie im Beisein der Schwester nicht hatte mitteilen wollen. Mir wurde deutlich, dass sich die APN-Pflegende von den anderen Pflegenden betreffend ihrer Kompetenzen unterschied. Sie arbeitete in der Zusammenarbeit mit anderen Berufs gruppen und wirkte als Pflegende auf mich sehr autonom. Sie traf in Zusammenarbeit mit ihren Kollegen Entscheidungen für die pflegerische Versorgung der Bewohner; Mitarbeiter fragten sie um pflegerischen Rat in spezifischen Pflegesituationen. Für einige Angehörige war sie die erste Ansprechperson, was sich daran zeigte, dass die Angehörigen sie um ein persönliches Gespräch baten, das pflegebezogen ausgerichtet war. Im Gespräch mit den Angehörigen konnte ich beobachten, dass sie mit ihnen Vereinbarungen traf, die sich nachhaltig auf die Pflegeplanung auswirkten.

Im Anschluss an diesen Vormittag gingen wir noch zu mehreren Bewohnern, mit denen wir ein Assessment durchführten. Dort konnte ich beobachten, dass sich die APN-Pflegende mit anderen Berufsgruppen traf, austauschte und dann wieder mit den Bewohnern zusammenkam. Ich konnte die APN-Pflegende einerseits in der direkten Pflege beobachten, andererseits aber auch in der Delegation verschiedener Aufgaben, während sie sich um organisatorische Abläufe kümmerte. Der Tag endete, indem wir ihre Rolle reflektierten und ich Informationen zur Lehrveranstaltung am Folgetag erhielt.

Am zweiten Tag trafen wir uns wieder in der Eingangshalle und gingen gemeinsam zum Seminarraum, wo bereits 12 Mitarbeiter aus unterschiedlichen Abteilungen auf uns warteten. Sie berichtete mir, dass sie einmal pro Woche zu einem jeweils unterschiedlichen Thema unterrichtete. An diesem Tag ging es um das Thema „Die Demenzerkrankung und die Erfassung von Schmerzen“. Sie stellte den Mitarbeitern zuerst das Krankheitsbild der Demenz vor und erläuterte anschließend den Umgang 
mit drei verschiedenen Assessmentinstrumenten, in denen das gezielte Fragen und das Beobachten der Bewohner eine besondere Bedeutung für die Einschätzung des Schmerzes hatten. Während sie die Theorie erklärte, bezog sie sich immer wieder auf die Bewohner im Hospital und stellte einen engen Theorie-Praxis-Transfer sicher, indem sie regelmäßig auf die Evidenz verschiedener Maßnahmen hinwies. In dieser Sequenz konnte ich die APN Pflegende deutlich in der Rolle der Lehrenden beobachten, in der sie die Mitarbeitern in ihrem Aufgabenbereich fachlich unterstützte und Möglichkeiten zu einer verbesserten, forschungsgestützten Pflegepraxis aufzeigte. Hier unterschied sie sich deutlich von den anderen Pflegenden, weil sie über eine Expertise in ihrem Feld verfügte, die im Vergleich zum Fachwissen der Mitarbeiter fortgeschritten war. Als Forschende, die neue Erkenntnisse in die Praxis trägt, zeigte sie, dass sie eigenständig ihren Aufgaben- und Verantwortungsbereich in der Begegnung von Bewohnern wahrnahm und somit für die Pflegenden eine wichtige Vorbildfunktion innehatte, deren sie sich bewusst war.

\section{Interview mit einer CNS-Pflegenden}

Im Rahmen meines Aufenthaltes in San Francisco war es mir neben dem WorkShadowing möglich, ein Interview mit einer CNS-Pflegenden mit dem Schwerpunkt kardiologische Pflegepraxis zu führen. Als CNS-Pflegende arbeitete sie seit einigen Jahren auf der medizinischen Abteilung des Krankenhauses der Universität von Kalifornien in San Francisco (UCSF). Anhand ihrer Schilderungen erfuhr ich, welchen Weg eine APN-Pflegende mit ihrer Rollenfindung gehen und wie sich eine Rollenentwicklung als APN-Pflegende gestalten kann.

Im Rahmen unseres Gespräches erinnerte sie sich an ihre Anfangszeit als CNS, die sie als schwierig erlebte. Sie fühlte sich zu Beginn in ihrer Rolle vielmals alleine, und der Austausch mit Berufskolleginnen in der täglichen Praxis trug entscheidend dazu bei, dass sie in die neuen Aufgabenfelder hineinwachsen konnte. Zu diesen neuen Aufgabenfeldern zählte vor allem der interdisziplinäre Austausch mit anderen Berufsgruppen, aber auch die intra- und interprofessionelle Zusammenarbeit. Sie berichtete davon, wie sie sich gegenüber anderen Berufsgruppen durchsetzen musste, was immer wieder mit einer Beschreibung ihrer eigenen Rolle einherging, die für viele KollegInnen in ihrem Umfeld neu war. In besonders schwierigen Situationen half ihr das Netzwerk, mit Herausforderungen umzugehen. Unter Netzwerk verstand sie nicht nur das persönliche Treffen mit APN-Pflegenden, sondern auch den Austausch mit ihnen über E-Mail, Facebook und Twitter. Als schwierig erlebte sie ebenfalls die Begleitung der Familien in Pflegesituationen, die neu für sie waren. Sie erinnerte sich, dass es herausfordernde Momente in der Begleitung der Familien gab, weil sie sich unsicher fühlte und über wenig Erfahrung im Koordinieren komplexer Aufgaben verfügte.

Zu Beginn ihrer Rolle hatten sehr viele Personen aus ihrem direkten beruflichen Umfeld hohe Erwartungen an ihre Rolle. Die Belastungen im Zusammenhang mit der neuen Berufsrolle erforderten eine klare Auseinandersetzung mit der eigenen Rolle. Gespräche mit der Führungsperson halfen ihr im Umgang mit ihren Aufgaben, aber auch der Kontakt $z u$ anderen CNS und das Erlernen eines neuen Zeitmanagements waren hilfreich für sie.

\section{Implementation einer APN-Rolle}

Welche Möglichkeiten bieten sich nun an, die Rollenentwicklung zur APN-Pflegenden zu erleichtern? Aus der Literatur kann abgeleitet werden, dass eine APN-Pflegende in Abgrenzung zu anderen professionellen Arbeitsfeldern sich mit ihrer Rolle und den dazugehörigen Aufgaben identifizieren muss. Dies inkludiert das Lernen einer speziellen Sprache, Fähigkeiten und Kenntnisse, welche Normen und Werte in sich tragen (Brykczynski 2009). Der Weg zur APN-Pflegenden kann nicht linear oder schrittweise beschrieben werden. Im Gegenteil, es handelt sich um einen Prozess, der einerseits mit einem Zuwachs an Expertise verbunden ist, andererseits aber Rückschläge bereithält, mit denen eine APN-Pflegende in den ersten Jahren ihrer Berufskarriere konfrontiert ist (Dreyfus and Dreyfus1986, 1996 in Brykczynski 2009). Somit sind APN-Pflegende besonders während und unmittelbar nach ihrer Ausbildung mit unterschiedlichen Stress- faktoren konfrontiert und mit Phasen, die zu einer Rollenbildung führen, die sowohl mit Frustrationen, als auch mit Gefühlen der Zufriedenheit einhergehen können (Brykczynski 2009).

Nach den Interpretationen von Powers (2009) sollte eine Pflegende versuchen, die Organisation mit ihren Abläufen zu erkennen und zu verstehen. Eine offene Kommunikation kann dazu beitragen, dass die veränderten Rollen in der Praxis zur Wirkung kommen. Dabei kommt der Identifikation mit dem eigenen Berufsfeld und der eigenen Biografie große Bedeutung zu. In den Interpretationen von Zafian (2009) wird betont, dass ohne eine angemessene Kommunikation innerhalb der Institutionen, sich Konflikte verhärten oder unausgesprochen bleiben können. Das kann dazu führen, dass besonders die neuen Rollen, die mit einer vertieften Pflegepraxis einhergehen, nicht gelebt werden können, was wiederum zur Verschwendung des Potenzials führen kann. Dabei kommt der jeweiligen Organisation eine wichtige Aufgabe zu, denn Organisationen sind in ihrer Arbeit dann effektiv, wenn sie professionelle Arbeit anstreben und ein hohes Maß an einheitlichem Verständnis in Bezug auf Werte sowie moralisches Kapital aufweisen (Mckeon 2006). So ist eine vertiefte Pflegepraxis (APN) eine Angelegenheit, für die eine Organisation zuständig ist, und folgerichtig muss sie sich auch darum kümmern, dass die APN-Pflegenden entsprechend integriert werden. Es kann sich als sinnvoll erweisen, Ziele mit der Führungsebene aufzustellen und zwar im Sinne der SMART-Regel:

(S) spezifisch,

(M) messbar,

(A) erreichbar(engl. accessible, achievable).

(R) realistisch und

(T) zeitgerecht (engl. time; Donahue 2007, Zafian 2009). Um sich zu integrieren, ist es unumgänglich, dass sich APNPflegende neben den Kontakten im Team auch an jene orientieren, die sie in der Praxis begleiten, das heißt pflegen. In eine Organisation mit der neuen Rolle hineinzuwachsen ist eine Herausforderung und kann als stressvoll eslebt werden. Im deutschsprachigen Raum ist die Rolle einer APN-Pflegenden noch relativ neu. Explizite 
Rollenbeschreibungen liegen bisher kaum vor, was es zusätzlich erschweren kann, herangetragene Erwartungen zu erfüllen. Nach den Erfahrungen, die bereits mit den Rollen einer CNS und einer NP gemacht werden konnten, lassen sich verschiedene Empfehlungen ableiten, die auch für eine APN-Pflegende im deutschsprachigen Raum gültig sein können ( $\rightarrow$ Infokasten).

\section{APN in der Schweiz}

Mittlerweile gibt es im deutschsprachigen Raum verschiedene Projekte, die das ANPKonzept und damit eine vertiefte Pflegepraxis zum Gegenstand ihrer Untersuchung gemacht haben (Spitz-Köberich u. SteinleFeser 2010, Zuñiga et al. 2010, Bläuer et al. 2011, Gaidys 2011, Imhof et al. 2011, Ullmann-Bremi et al. 2011, Ulrich et al. 2011). Aktuell sind das beispielsweise Publikationen, welche die APN-Praxis im häuslichen Pflegesetting untersucht haben. Einerseits, um die Gesundheit alter Menschen zu erhalten und damit einen Heimaufenthalt zu verhindern oder hinauszuzögern (Imhof et al. 2011), andererseits konnte beispielsweise ein multidisziplinäres pflegegeleitetes Edukationsprogramm zur stationären Betreuung von Patientinnen und Patienten mit einer Herzinsuffizienz die Patientenversorgung optimieren (Bläuer et al. 2011). Im Vergleich zu Kalifornien fehlt unseren Masterabsolventen in der Schweiz allerdings sowohl eine Lizenzierung der Bezeichnung APN, als auch ein Verordnungsrecht obwohl der Bedarf an professioneller Pflegepraxis laut statistischer Zahlen auch in Zukunft steigen wird. Damit geht nicht nur ein Zuwachs von Menschen mit chronischen Erkrankungen einher; wir begegnen heute eine Vielzahl an informierten Patienten, die eine professionelle Unterstützung innerhalb des Gesundheitssystems erwarten. Aktuell finden diesbezüglich innerhalb der Schweiz Diskussionen statt, u.a. im Januar 2012, zu der die ZHAW Alba DiCenso zu einem Vortrag und einer anschließenden Podiumsdiskussion eingeladen hat. Sie ist seit 2001 Leiterin APN der "Canadian Health Service Research Foundation" (CHSRF) und des "Canadian Institute of Health Research" (CIHR). Sie beschäftigt sich bereits seit vielen Jahren im Rahmen ihrer Forschung mit der Einführung von
APN, sowie den beiden Rollen der CNS und NP die auch in Kanada sehr bekannt sind.

\section{Master of Science in Pflege}

Im deutschsprachigen Raum bestand seit dem Jahr 2000 erstmalig die Möglichkeit, mit dem ersten Studiengang der Pflegewissenschaft einen Master of Science in Pflege mit Fokus auf APN zu absolvieren. Seit dem Herbst 2010 bietet auch die Zürcher Hochschule für Angewandte Wissenschaften (ZHAW) in Kooperation mit der St. Galler Hochschule für Angewandte Wissenschaften (FHS) und der Berner Fachhochschule (BFH) die Möglichkeit, einen dreisemestrigen konsekutiven Master of Science in Pflege (MScN in Pflege) mit dem Schwerpunkt Advanced Practice Nursing an (Teilzeit: sechs Semester). An der ZHAW liegt der Schwerpunkt auf der Pflege von Menschen mit akut kritischen Gesundheitsproblemen im stationären Bereich und der Pflege von Menschen mit akuten und chronischen Gesundheitsproblemen in der gemeindenahen und häuslichen Versorgung. Studierende setzen sich in diesem Rahmen beispielsweise auseinander mit Interventionen, die zur
Unterstützung des Selbstmanagements beitragen, um Klienten in einem unabhängigen Leben zu unterstützen. Andererseits erwerben sie im Bereich der Akutversorgung, beispeilsweise in Interventionen zum Symptommanagement und der Palliation, Kompetenzen. Der Studiengang an der ZHAW ist eng mit dem Forschungsbereich des Instituts für Pflege verbunden, was es ermöglicht, die Theorie an praktischen Beispielen aus der Forschung zu erlernen und Bezüge zu aktuellen Projekten herzustellen. Das Ziel des Studiengangs MScN in Pflege ist es, primär Pflegeexpertinnen und Pflegeexperten APN auszubilden, die eine spezialisierte Versorgung von bestimmten Patientengruppen innerhalb verschiedener Settings anbieten. Die inter- sowie intradisziplinäre Zusammenarbeit hat einen hohen Stellenwert; so gehört zu den Ausbildungszielen, dass Studierende sich auf verschiedene Beratungssituationen innerhalb der Prävention und Gesundheitsförderung mit anderen Berufsgruppen vorbereiten. Pflegefachpersonen haben mit diesem Studium die Möglichkeit, sich einer vertieften Pflegepraxis zu widmen und Schritte ei-

\section{EMPFEHLUNGEN}

Integration einer APN-Rolle in die Pflegepraxis

1. Sei pro-aktiv: Sage, was du brauchst, um dich orientieren zu kõnnen.

2. Finde einen Mentor oder orientiere dich im Feld einer/s anderen APNPflegenden.

3. Informiere dich über die Kunden und helfe ihnen, sich wohl zu fühlen.

4. Bilde ein professionelles Netzwerk

5. Nimm dir regelmäßig Zeit für die Reflexion der eigenen Rolle.

6. Pflege eine vertrauensvolle Beziehung zu Mitarbeitern und Kunden

7. Stelle eine gute Patientenversorgung sicher und achte auf Ergebnısqualität

8. Entwickele mit der informellen und formellen Führungsebene Leitlinien für eine gute Kommunikation.

9. Triff Entscheidungen und strebe eine gute Zusammenarbeit mit allen an der Pflege Beteiligten an.
10. Sei dir bewusst, dass du mit deiner Rolle ein Vorbild in der Organisation darstelist und handle im Sinne einer professionellen Pflege.

11. Bilde in der Organisation Kleingruppen, die sich Themenbereichen widmen und begleite sie.

12. Kommuniziere deine Funktion und die jeweiligen Kompetenzen, die mit den Rollen in deiner Funktion verbunden sind.

13. Suche nach Möglichkeiten, dich mit Kolleginnen und Kollegen auszutauschen und mache deine Rolle anderen gegenüber transparent.

14. Arbeite auf den Abteilungen und sei präsent.

Quelle: modifiziert in Anlehnung an Powers 2009 und Zafian 2009 


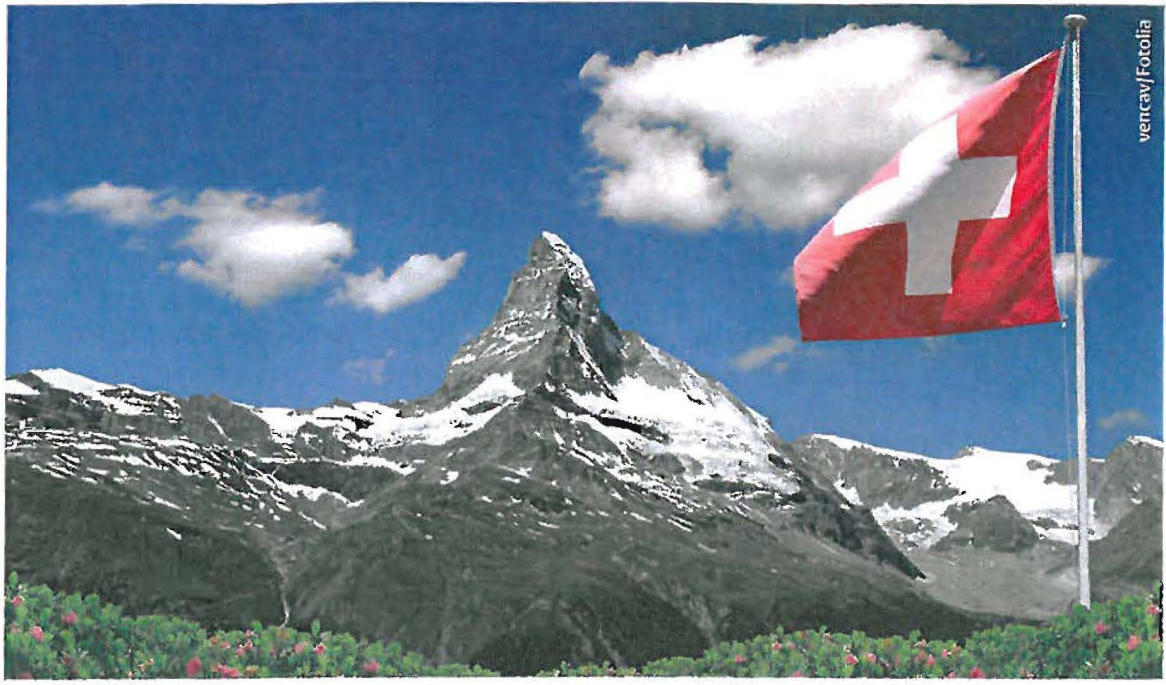

ner persönlichen Karriereplanung in der direkten Pflege zu tätigen. Im Hinblick auf die demografische Entwicklung und der Zunahme an älteren Menschen sowie chronischen Erkrankungen, wird es notwendig, dass APN-Pflegende die pflegerische Versorgung übernehmen und dazu neben erweiterten pflegerischen Wissen und Können auch erweiterte Kompetenzen und Verantwortlichkeiten erhalten.

\section{Persönliches Fazit}

Bei meinem Besuch in Kalifornien habe ich anhand von Beispielen sehen können, dass die Rolle einer APN Pflegenden im Vergleich zum deutschsprachigen Raum fest implementiert ist. Während der Begleitung der APN in ihrem Berufsalltag im Krankenhaus mit dem Schwerpunkt Rehabilitation war es mir nicht möglich, eine klare Rollenunterscheidung zwischen CNS und NP $2 u$ sehen. Ich konnte jedoch die Umsetzung der Kompetenzen einer APN in ihrem Aufgabenfeld beobachten. Die Reise nach Amerika hat mein Bewusstsein für die Spezialisierungen und Erweiterung der Pflegepraxis verstärkt. Ich konnte sehen, wie sich Pflege deutlich in ihrer Profession positioniert und gesellschaftlich legitimiert ist. Eindrücklich fand ich, wie selbstverständlich die Integration verschiedener Kulturen war; Abteilungen, die ausschließlich auf eine Kultur spezialisiert waren, hatte ich zuvor noch nicht gesehen. Auf dieser Reise habe ich viele gute Gespräche führen und verschiedene Eindrücke in die Pflegepraxis gewinnen können, was mich darin bestärkt, mich weiterhin für eine professionelle Pflegepraxis einzusetzen.

\section{Danke!}

Ein herzlicher Dank geht an Heidi Longerich ( $\mathrm{RN}, \mathrm{MScN}$ ), Leiterin des Instituts für Pflege an der ZHAW, die diesen Auslandsaufenthalt ermöglichte. Ein besonderer Dank geht an die Leiterin des Studiengangs Master of Science in Nursing an der ZHAW; Prof. Dr. Romy Mahrer Imhof ( $\mathrm{PhD}, \mathrm{RN}, \mathrm{FESC})$. Sie stellte wichtige Kontakte bereit und ermutigte mich, diese Reise $z u$ unternehmen.

\section{Literatur}

1 Bläuer, C., Mahrer Imhof, R., Brunner-La Rocca, H.، Müller, C., Eze, C., Milbich, L., Spirig, R. (2011): Entwicklung und Implementierung eines multidiziplinären pflegegeleiteten Programms zur stationären Betreuung von Patientinnen und Patienten mit Herzinsuffizienz: Das Basler HI-Programm. Pflege; 24(1): 29-41

2 Canadian Nurses Association. (2008). Advanced nursing practice: A national framework. Ottawa, ON. Canadian Nurses Association. Retrieved January 12th, 2009, http:// www.srna.org/images/stories/pdfs/nurse_resources/2009_ advanced_NP.pdf (04.12.2013)

3 Delamaire, M., Lafortune, G. (2010): "Nurses in Advanced Roles: A Description and Evaluation of Experiences in 12 Devejoped Countries", OECD Health Working Papers, No. 54, OECD Publisting from http://dx.doi. org/10.1787/5kmbrcfms5g7-en (04.12.2011)

4 Donahue, G. (2007): Top achievement: Creating S.M.A.R.T goals, from http://www.topachievement.com/ smart.html (30.12.2011)

5 Gaidys, U. (2011): Qualität braucht Kompetenz und Verantwortung - Herausforderungen und Perspekriven einer Advanced Nursing Practice für die Gesundheitsversorgung aus pflegewissenschafticher Sicht. Pflege: (24) 1: 15-20 6 Hamric, A., Spross, J., Hanson, C. M. (2009). Advanced Practice Nursing: An Integrative Approach. $4^{\text {th }}$ ed. St. Louis, MO: Elsevier Saunders

7 Imhof, L., Naef, R., Mahrer Imhof, R., Petry, H. (2011): SpitexPlus: Assessment und fortgeschrittene Pflegeinterventionen für zuhause lebende alte Menschen und ihre Familien. Pflege; $24(1): 43-56$
8 Keeling A. W: A Brief History of Advanced Practice Nursing in the United States In: Hamric. J. Spross, C M. Hanson (2009). Advanced Practice Nursing: An Integrative Approach. $4^{\text {th }}$ ed. St. Louis, MO: Elsevier Saunders 9 McKeon, L.O. (2006): Safeguarding paciencs, complexity science, high reliability organizations and implications for team training in healthcare. Clinical Nurse Specialist. 20(6), 298-304

10 Spitz-Köberich, C., Steinle-Feser, B. (2010): Ein Jahr Advanced Nursing Practice in der Kinderkardiologie - ein erster Rückblick Pflege; (23) 6: 411-416

11 Peplau, H. (1965) Specialization in professional nursing. Nursing Science, 2: 268-287

12 Powers, J.: Finding a Place in the Organization. In: $M$ Duffy, S. Dresser, J S. Fulton: Clinical nurse specialist toolkit: a guide for the new clinical nurse specialist (2009): 23-30

13 Ullmann-Bremi, A, Schlüer, A-B., Finkbeiner, G. Huber, X. (2011): aWie ein ANP-Team laufen lernt» - Herausforderungen und Chancen von ANP-Teams am Universitätskjnderspital Zürich. Pflege; (24) 1: 21-28 14 Ulrich, A., Hellstern, P., Kressig, R W., Eze, G., Spirig. $R$ (2010): Advanced Nursing Practice (ANP) im direkten Pflegealltag: Die pflegerische Praxisentwicklung eines akutgeriatrischen ANP-Teams. Pflege: (23) 6: 403-410 15 Zafian, R. B.: Learning the Ropes: Orientation. In: M. Duffy, S. Dresser, J S Fulton: Clinical nurse specialist toolkit: a guide for the new clinical nurse specialist (2009): 43-51

16 Zúñiga, F. Jenni, G., Wiesli, U., Schwendimann. R. (2010): Entwicklung der Rolle der Advanced Practice Nurse in der stationären Langzeitpflege älterer Menschen in der Schweiz. Pflege; (23) 6: 375-383

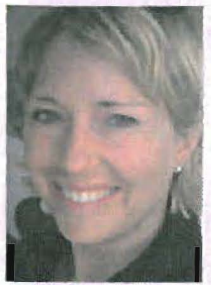

Or. Daniela Händler-

Schuster

leitet an der Zürcher Hochschule für Angewandte Wissenschaften (ZHAW) als Dozentin im Studiengang MSCN das Modul Forschungsmethoden II und arbeitet als wissenschaftliche

Mitarbeiterin im Institut für Forschung und Entwicklung. Ihre pflegerische Expertise liegt in der heimbezogenen und gemeindenahen Langzeitpflege mit dem Schwerpunkt der pfiegerischen Betreuung von Menschen mit Demenz

Korrespondenzadresse

daniela.haendler-schuster@zhaw.ch

\section{Prof. Dr. Romy Mahrer-Imhof}

leitet an der ZHAW den Studiengang Master of Science in Pflege und ist verantwortlich für das Modul APN. Sie hat die Professur für Familienzentrierte Pflege inne und forscht zu Erkrankungen und deren Auswirkungen auf familiale Systeme.

Korrespondenzadresse

romy.mahser@zhaw.ch

\section{Bibliografie}

DO! 10.1055/s-0032-1327016

Psych Pflege 2012; 18: 252-257

(Q) Georg Thieme Verlag KG

Stuttgart - New York - ISSN 0949-1619 